\title{
LOS HERBARIOS DE LAS EXPEDICIONES CIENTÍFICAS ESPAÑOLAS AL NUEVO MUNDO
}

\section{Paloma Blanco Fernández de Caleya}

\section{RESUMEN}

Los herbarios confeccionados por las expediciones botánicas españolas al Nuevo Mundo durante el siglo XVIII constituyen uno de los tesoros más importantes del Real Jardín Botánico de Madrid. Estas colecciones de plantas secas documentan la existencia de las especies en el tiempo y en el espacio, son recursos esenciales para la investigación botánica. Los ejemplares tipo y de referencia de estas colecciones son fundamentales para la exacta identificación de cada especie y sus variedades. Sirven para dar validez a la investigación botánica asegurando que se pueda repetir o comparar con investigaciones futuras.

\section{SUMMARY}

The herbaria compounded by the botanical Spanish expeditions to the New World during the XVIIIth Century constitutes one of the most important treasures of the Royal Botanical Garden from Madrid. These collections of dry plants document the existence of the species in time and in space, and they are essential resources for the botanical investigation. The specimens type and of reference of these collections are fundamental for the 


\title{
PALOMA BLANCO FERNÁNDEZ DE CALEYA
}

\begin{abstract}
correct identification of each species and their varieties. They are used as to give validity to the botanical investigation securing that one could repeat or compare with future investigations.
\end{abstract}

\section{Introducción}

Siempre que hablamos del Real Jardín Botánico de Madrid destacamos sus colecciones de plantas secas, sus herbarios, y todos los documentos en donde se hacen referencia a dichas plantas. Entre los tesoros más valiosos se encuentran las plantas traídas del Nuevo Mundo gracias a las expediciones científicas, motivadas por la política ilustrada del siglo XVIII.

Estas colecciones de plantas secas o "herbarios» forman parte del Patrimonio Histórico Español que como se dice en el Preámbulo de la Ley 16/1985, de 25 de junio, sobre dicho Patrimonio «son el principal testigo de la contribución histórica de los españoles a la civilización universal y de su capacidad creativa contemporánea».

Hoy día se tiende a valorar estas colecciones cuantificando lo que se haya publicado sobre ellas. En relación a los herbarios se valora el número de "tipos" que contiene la colección; estos son los ejemplares originales utilizados por los botánicos para dar nombre a las distintas especies. El nombre que se debe utilizar para nombrarlas es el que primero se publicó correctamente. Cuanto mayor sea el número de tipos y mayor el área recolectada, más rica es la colección.

En este sentido, los resultados de nuestras expediciones del siglo XVIII al Nuevo Mundo no son tan buenos como hubieran podido ser. Gran parte del material allí recolectado, original entonces si se hubiera publicado, quedó inédito o se publicó a destiempo aumentando la lista de sinonimias; parte de este material fue publicado por botánicos extranjeros, muchas veces sin indicar su procedencia. Por otro lado, los botánicos tendían a acrecentar la colección y el estudio de ella con la mayor variedad posible de especies, pero tenían poco interés en recolectar de nuevo las mismas especies en distintas localidades; además, al estar aquellos territorios poco poblados, los expedicionarios a menudo no sabían exactamente donde estaban, pocos ejemplares incluyen etiquetas con información completa y concreta de la localidad donde fueron colectados.

Pero aún así es un éxito que, a pesar de tantos contratiempos, podamos hoy disponer de unas colecciones en muy buen estado de conserva- 
ción. Junto a los datos asociados a ellas, dibujos, descripciones, cartas, etc. documentan la existencia de especies en aquel tiempo y en aquellas extensas tierras, y posibilitan nuevos estudios y descubrimientos a través de proyectos de educación e investigación.

\section{Un poco de historia sobre el origen de estos herbarios}

En 1492 Cristóbal Colón hizo su primer viaje de descubrimiento y exploración y volvió de América cargado con productos nativos de las nuevas tierras. Lo que allí se encontró, revolucionó el saber, causando una profunda crisis en la filosofía y ciencia del Renacimiento europeo. A partir de entonces la observación y la experiencia como fundamento de la razón fueron las bases del pensamiento científico de los nuevos exploradores en América. Este Descubrimiento se ha considerado como el inicio de un fuerte vínculo entre las culturas del Viejo y Nuevo Mundo, pero también como el primer viaje de recolección para un museo ${ }^{1}$.

En el último tercio del siguiente siglo, 1570-1577, es cuando Felipe II envía una Expedición botánica al Virreinato de Nueva España bajo la dirección de su médico Francisco Hernández, nacido en 1517. Distinguido por sus conocimientos botánicos, Hernández, fue el encargado de hacer un inventario de los productos naturales de aquellas tierras. Allí colectó plantas y acumuló diversa información sobre la medicina azteca desarrollada en aquella época. Su obra, el estudio metódico de los recursos naturales de aquel país, se agrupó en diez y siete (16?) tomos en folio entre texto y láminas, que se enviaron a España junto con las plantas secas y colecciones de semillas mexicanas para cultivar en los jardines de Aranjuez (primer jardín botánico creado en España por consejo de Andrés Laguna a Felipe II). La muerte de Hernández en 1578, impidió que su obra se llegara a publicar entonces, quedando depositada en el biblioteca de El Escorial. El primer herbario magnífico con el que hubiéramos podido contar era el de Hernández, pero un desgraciado fuego en 1671 en El Escorial, fue la causa de su desaparición. Aún así parte de su obra fue publicada posteriormente gracias a Ximenez (1615) en Méjico, Reccho (1651) en Roma y Gómez Ortega (1790) en

\footnotetext{
1 Palacios, F. et al. eds. (1992), «Resoluciones...», Int. Symp. \& First World Congress on Preserv. and Conserv. of Nat. Hist. Col. 1, 25-32, Madrid, p. 31.
} 
Madrid; lo que sin duda repercutió entre los naturalistas europeos en los siguientes siglos ${ }^{2}$.

Dos siglos más tarde, a mitad del siglo XVIII, gracias a que Linneo (1707-1778) desarrolló un sistema de nomenclatura binario, los seres vivos pudieron ser nombrados y ordenados con mayor facilidad que antes. A partir de entonces muchas personas se interesaron en acumular colecciones y clasificar los organismos. Esto supuso en la Ciencia un avance importantísimo, al pasar los Museos y Jardines Botánicos de ser meras colecciones de curiosidades a centros más prácticos de desarrollo del conocimiento científico.

Este momento coincidió en la Península con un claro apoyo a las actividades científicas y técnicas por parte del poder real en pro de la Ilustración ${ }^{3}$.

Aunque Linneo había criticado duramente a los botánicos españoles, Fernando VI le propuso aceptar un puesto digno en España o la designación de un discípulo instruido en las nuevas doctrinas y con dominio del conocimiento de las plantas. Pehr Löfling fue el naturalista elegido por Linneo, que vino a la Península en 1751, llegando a Madrid procedente de Oporto en donde había desembarcado 4 .

Aprovechando la venida de Löfling a la Península; Fernando VI dispuso que en la Expedición de Límites al Orinoco (1753-1761), comandada por José de Iturriaga, que tenía como objetivos fijar los límites en el norte de Venezuela, luchar contra el contrabando y contener a los holandeses, se añadiese el estudiar la naturaleza de aquellos territorios, tanto por su interés estratégico y comercial (quina, cacao y canela), como el estrictamente científico; a Löfling le encargó este estudio. Desgraciadamente la muerte en 1756 del sueco, a los dos años de su llegada a América, frustró los planes. Fernando VI murió en 1759 y el nuevo rey Carlos III dio por concluida en 1761 la Expedición. Los papeles, láminas y herbarios a la muerte de Löfling fueron enviados por José Iturriaga a Madrid, donde pasaron por diversas manos. El herbario se perdió, así como la mayoría de los originales de las láminas botánicas. Linneo intentó conseguir copia

\footnotetext{
2 Colmeiro, M. (1858). La Botánica y los botánicos de la Península hispano-lusitana, 1-216. Madrid, p. 154.

3 PUIG-SAMPER, M.A. (1991), Las expediciones científicas durante el siglo XVIII, ed. Akal, Madrid, 28, 1-54, p. 23.

4 Colmeiro, M. (1875), «Bosquejo histórico y estadístico del Jardín Botánico de Madrid». Anales Soc. Esp. Hist. Nat. 4, 241-345, p. 217.
} 
de los manuscritos nada más conocer la muerte de su discípulo y lo consiguió gracias al capellán de la legación sueca en Madrid, aunque no tuvo la misma suerte con los duplicados del herbario recolectado. A pesar de todo, con la correspondencia que de Löfling tenía, formó el Iter Hispanicum, escrito en sueco y en donde están descritas unas mil trescientas plantas españolas, aunque pocas de las americanas ${ }^{5}$.

Un año después de haberse embarcado Löfling para América al servicio de España, Fernando VI, por Real Orden de 21 de octubre de 1755, dispuso que en su huerta del Soto Migascalientes se hiciera un jardín botánico para cultivar las plantas del jardín de José Quer (1695-1764). Este, nombrado primer profesor en 1757, fue el que restauró y enseñó la Botánica en Madrid, aunque su fidelidad a Tournefort le impidió progresar con los principios de Linneo ${ }^{6}$.

Más tarde, Miguel Barnades, sucesor de Quer, publicó en 1767 unos Principios de Botánica, obra elemental para la enseñanza de esta ciencia con la que intentó facilitar y generalizar su conocimiento, único medio de aumentar el número de buenos cooperadores con el objeto de reunir materiales para escribir una Flora Española.

Las plantas americanas que al principio llamaron tanto la atención de los españoles estuvieron mucho tiempo olvidadas. Al mostrar interés por ellas los botánicos extranjeros, despertaron las inquietudes de los botánicos españoles entonces preocupados por estudiar las de la Península, pues pensaron que tan españoles debían ser los que estudiasen unas como las otras. Estas ideas también fueron las del Gobierno comenzando así una nueva era para la botánica española.

El apoyo del poder real, plasmado con la creación de Gabinetes, Museos y Jardines Botánicos por España y sus colonias, unido a los esfuerzos de los científicos de entonces, posibilitó la organización de varias expediciones de investigación de la naturaleza, para examinar los territorios de los dominios españoles, y cuales de sus recursos eran aprovechables.

\footnotetext{
${ }^{5}$ Colmeiro, M. (1842). Ensayo histórico sobre los progresos de la Botánica desde su origen hasta el día, considerados más especialmente con relación a España, 1-71. Barcelona, p. 28. PUIG-SAMPER, M. A. (1991), pp. 24-27. MuÑoz-GARMEDIA, F. (1993), «Diarios y trabajos botánicos de Luis Neé», en La Expedición Malaespina 1789-1794, 3, 1-409, pp. 19-21.

6 Colmeiro, M. (1858), p. 163.
} 


\section{PALOMA BLANCO FERNÁNDEZ DE CALEYA}

A la muerte de Barnades en 1771, ocupó la plaza de primer catedrático en el Jardín de Migascalientes Casimiro Gómez Ortega (1740-1818). La influencia que ejerció en pro de la Botánica cabe ser destacada en la historia de la Botánica ${ }^{7}$. En 1781 se trasladó el Jardín de Migascalientes al Prado, en donde nada se escatimó para «dar a la ciencia toda la ostentación de que es digna y con el objeto de que no desmereciese en ninguna otra cosa de los demás jardines de Europa». Gracias a Gómez Ortega se formaron muchos botánicos en esta época, que luego fueron los que ocuparon cátedras y dirigieron distintos jardines botánicos tanto en la Península como en los territorios españoles de ultramar, a donde habían llegado por distintos motivos; algunos de ellos fueron posteriormente reconocidos como miembros de las distintas expediciones científicas españolas. Uno de los frutos de estas expediciones botánicas son los herbarios que trataremos a continuación.

\section{La Expedición al Perú y Chile}

Al aceptar el gobierno español la propuesta francesa de exploración del virreinato del Perú, se originó la Expedición Botánica al Virreinato de Perú y Chile (1777-1788), pero con la condición de que la dirección estuviera a cargo de los españoles, medida adoptada tras la experiencia anterior con la expedición de La Condamine, y con la idea de aprovechar la mayor formación de los científicos franceses. Fueron nombrados Hipólito Ruiz (1754-1816), y José Pavón (1754-1840), como primer y segundo botánico respectivamente, Joseph Dombey (1742-1794), «miembro acompañante» y Joseph Brunete e Isidro Gálvez, como dibujantes.

Durante el primer año 1778-1779 herborizaron juntos Dombey, Ruiz y Pavón en los alrededores de Lima y en las provincias cercanas del litoral, desplazándose hacia el norte hasta Chancay y Haura. Estos materiales fueron enviados a España en marzo de 1779 como primera muestra del trabajo; eran «esqueletos», plantas secas, de 300 especies diferentes y 242 dibujos $^{8}$. En 1779 Ruiz entró en la región andina y llegó a Tarma, mientras Dombey hacía otro itinerario hacia Cheuchin. En 1780 salieron hacia

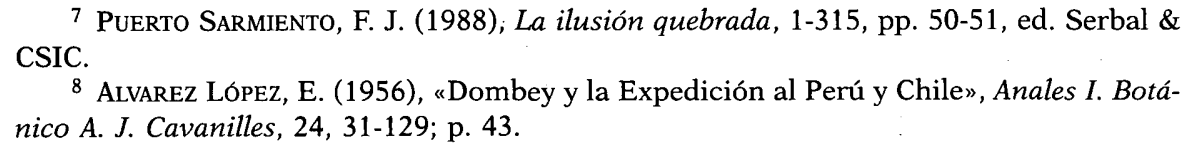

${ }^{8}$ Alvarez López, E. (1956), "Dombey y la Expedición al Perú y Chile», Anales I. Botánico A. J. Cavanilles, 24, 31-129; p. 43. 
Huánuco, región situada sobre la vertiente este del ramal central de la cordillera y la verdadera entrada a la amazonía. La privilegiada situación de esta ciudad y su riqueza florística la convirtieron en el centro principal de la expedición ${ }^{9}$. Aquí se encontró por primera vez el árbol de la Quina. De regreso a Lima a comienzos de 1781 hicieron nuevos envíos a España e iniciaron una nueva entrada a Chancay. Debido a los conflictos sociales provocados por el levantamiento de Túpac Amurac, se cambiaron los planes, decidiendo el viaje hacia Chile. Desde su llegada a Talcahuano, en enero de 1782, exploraron Concepción, la cordillera andina y los alrededores de Santiago y Valparaíso. Pasado un año regresaron al Puerto de El Callao. Parte de estos materiales recolectados en Chile se perdieron en un naufragio. En 1784 el botánico francés Dombey se volvió a su país ${ }^{10}$, mientras que los botánicos españoles volvieron a Huánuco para seguir estudiando a fondo aquella zona de quinas. Aquí se sumaron el farmacéutico navarro Juan Tafalla y el dibujante Francisco Pulgar. En 1785 perdieron parte de sus colecciones en un incendio cerca de Macora. En 1787 se recibió la orden de volver a la península. Una vez retirados los responsables iniciales de la expedición, Tafalla y Pulgar, junto con el botánico Juan Manzanilla, siguieron explorando las regiones peruanas y organizaron entre 1799 y 1808 varias expediciones por Guayaquil y Quito que luego dieron lugar a la Flora Huayaquilensis ${ }^{11}$. Para la publicación de su Flora, Ruiz y Pavón completaron en Madrid sus observaciones de

\footnotetext{
9 Estrella, E. (1988), "La Expedición Botánica en el Virreinato del Perú» en $L a$ Expedición Botánica en el Virreinato del Perú, Madrid y Barcelona, pp. 52-54.

10 Alvarez López, E. (1956), pp. 127-129. En la partición de las colecciones traídas por Dombey a su regreso a Cádiz, al final hubo cumplimiento de lo establecido antes de la partida de la expedición y consignado en las "Instrucciones» que la regían. El compromiso prohibía a Dombey la publicación de sus manuscritos antes del retorno de los expedicionarios españoles y también le comprometía a entregar a su vuelta para el Rey de España una colección igual a la que se enviase a Francia.

Aunque fue admitido de facto y de jure, en el envío de plantas que se trajeron en el navío El Buen Consejo, que salió en abril de 1779 del Puerto del Callao al de Cádiz, el reparto que hizo de las plantas fue notoriamente desigual y desfavorable para las que se quedaron en España, por lo que fue amonestado. Alvarez LóPEZ, op. cit., p. 70. Aún así Dombey intentó al terminar su estancia en Perú llevarse entera sú colección, justificándose al haber intercambiado plantas con Ruiz y Pavón mientras se encontraba en el Perú. Aunque no tuvieron ningún éxito sus pretensiones, los franceses convirtieron su obligación en un obsequio hacia España.

11 Estrella, E. (1989), «Introducción histórica: La Expedición de Juan Tafalla a la Real Audiencia de Quito (1799-1808)» en La Flora Huayaquilensis, vol. I, XII-CVI, Madrid.
} 


\section{PALOMA BLANCO FERNÁNDEZ DE CALEYA}

Perú y Chile, cultivando sus semillas y observando las plantas que crecían junto a las que seguía enviando Tafalla.

Es la expedición que tuvo mejor suerte, ya que volvieron a la Península cuando todavía Gómez Ortega tenía posibilidades de publicar sus resultados. En 1792, vio la luz la Quinología de Ruiz; en 1801, el Suplemento a la Quinología de Ruiz y Pavón. Sabiendo lo complejo que era editar una obra de las características de la Flora del Perú y Chile, quisieron previamente dar a conocer sus novedades en Prodromus Florae Peruvianae et Chilense Previo (1794), y en Systema vegetabilium Florae Peruvianae et Chilensis (1798). Desde 1798 hasta 1802 pudieron emprender la publicación de la Flora Peruviana y Chilensis. Estas obras son punto de referencia obligado para todos los estudios taxonómicos de aquella flora; en ella llegaron a descubrir 1.612 nuevas especies, lo que hizo que inmediatamente dieran valor a su colección de plantas secas, sus herbarios, pues contienen los patrones para la aplicación de esos nombres.

Hacia el año 1814 ya no había financiación para la Flora. Pavón sin ayuda del gobierno y desesperado por la falta de dinero, ofreció plantas de su herbario a J. E. Smith. Este le puso en relación con Aylmer Bourke Lambert, botánico británico y colector de considerable prestigio ${ }^{12}$ en 1814 comenzaron las negociaciones al recibir Pavón una carta, el 28 de mayo, de éste que pedía duplicados de herbario, insectos, y otros ejemplares de historia natural para comprar. La correspondencia entre Pavón y Lambert que duró los siguientes 11 años, "Letters A. B. Lambert Esq.», se encuentra en la biblioteca del Real Jardín Botánico de Kew.

El 12 de agosto de 1814 Pavón escribió a Lambert, ofreciéndole 1.500 plantas americanas, cada una con su etiqueta. Parece ser que este primer envío no se llegó a realizar hasta 1816. Lambert lás recibió con entusiasmo en julio. Durante los años siguientes el comercio continuó, Pavón enviaba ejemplares de herbario, muestras de madera, cortezas y conchas de mar. Lambert correspondía con dinero y con las publicaciones botánicas necesitadas por Pavón, que se encontraba aislado de la comunidad botánica internacional. Parece desprenderse de las cartas que Lambert recibió de Pavón, entre 1816 y 1820 , por lo menos 6.000 , o hasta 10.000 plantas, la mayoría de América pero incluyendo algunas de España. Las cartas de Pavón no siempre aclaraban de que envío se trataba y cuantas

\footnotetext{
12 Miller, H. S. (1979), "The herbarium of Aylmer Bourke Lambert. Notes on its acquisition, dipsersal, and present whereabouts», Taxon, 19, pp. 489-656.
} 
plantas contenían. Después de 1820 el comercio con Lambert fue disminuyendo hasta desaparecer. La última carta de Pavón, que se encuentra en Kew, está fechada el 18 de julio de 1825. Pavón tenía más de 70 años y seguía necesitando dinero. Negoció durante 1825 y 1826 con A.P. de Candolle, sin éxito, aunque incluso le hizo mejor oferta que a Lambert. Mejor suerte tuvo al vender ejemplares a otros botánicos europeos como a Moise Etienne Moricand (1779-1854). Este comentó en una carta a su amigo De Candolle, el 8 de septiembre de 1827, haber recibido 800 ejemplares de Pavón.

Afortunadamente para Pavón, también pudo establecer un sospechoso negocio con otro rico colector británico, Philip Barker Webb (17931854). Ello está documentado en una serie de cartas, «la correspondencia de Webb», del Instituto Botánico de Florencia ${ }^{13}$. Según parece, Webb llegó a recibir 4.994 ejemplares, incluyendo 2.345 de Nueva España; 2.048 de Perú, 270 de Guayaquil y Quito; 142 de Chile, 56 de Cuba; 34 de Puerto Rico; 11 de Bahía Botánica; 11 de Filipinas; 3 de Sinus Hudsonis; 3 de Islas 3as, etc... Los envíos de Pavón no sólo a Webb sino a Lambert, a menudo incluían uno o varios ejemplares de la misma colección. En una carta a Webb (29 de enero de 1828) enumeró un total de 2.684 «especies»; 243 duplicados; 60 triplicados, 6 quadriplicados y 1 "multiplicado» ${ }^{14,15}$. Muchas de estas plantas que Pavón vendió a Webb permanecen en Florencia todavía sin ser estudiadas.

Aunque los ejemplares que Pavón vendió habían sido colectados durante las expediciones patrocinadas por el gobierno español, y claramente pertenecían al Estado y no a Pavón, nadie se preocupó bastante de ello y al estar este material en manos de Pavón y no seguros bajo el control del Jardín Botánico, como estuvo la colección de Mutis, pudieron salir fuera de España sin posibilidades de recuperación. Gracias a ello, botánicos de otros países pronto empezaron a saber de su existencia.

\footnotetext{
13 Pichi-Sermolli, R. (1949), «Le collezioni cedute da J. Pavón a F. B. Webb e conservate nell'Herbarium Webbianum», Nuovo Giornale Botanico Italiano, 56 (4), pp. 699 701.

14 McVaugh, R. (1990), "Botanical results of the Sessé \& Mociño Expedition (17871803). IV. The Library and the Herbarium of the Expedition". Contr. Univ. Mich. Herb., 17, 183-214, p. 197.

15 McVaugh, R. (1987), "Botanical results of the Sessé \& Mociño Expedition (17871803). III. The impact of this and other Expeditions on contemporary Botany in Europe». Contr. Univ. Mich. Herb., 16, 155-171, pp. 168-169.
} 
Los ejemplares de la colección de Moricand y especialmente los que Pavón vendió a Lambert, tuvieron un considerable impacto en la botánica del siglo XIX. Más de trescientos taxa se basaron en estos duplicados. Los botánicos implicados en esta actividad fueron los botánicos eminentes de la época: Baillon, Bentham, Boissier, Brongniart, A. de Candolle, Cassini, Choisy, David Don, George Don, Decaisne, Duchartre, Dunal, A. de Jussieu, Lindley, Nees, Müller Argoviensis, Reichenbach filius.

Con el herbario de Lambert hubo unos cuantos problemas, pues se vendió en 1842 en una subasta pública, en muchos lotes separados. Las partidas más grandes fueron revendidas por comerciantes a colectores. Pocos botánicos estaban entre los compradores; la única notable excepción fue Robert Brown, quien compró para el British Museum un lote grande que, según parece, incluye 665 especímenes de plantas mexicanas entre otras cosas vendidas por Pavón a Lambert ${ }^{16}$. Una larga serie del herbario de H.B. Fielding, originalmente de Lambert, está en la Universidad de Oxford (OXF). En el herbario de Berlin-Dahlem hay pliegos de Ruiz y Pavón que llegaron en diferentes años por diferentes conductos, uno de estos lotes es de 1.620 especies de las adquiridas por Lambert y que llegaron a Berlín gracias a Klotzsch en 1893; parte se envió a la Smithsonian Institution (US). Según Miller, op. cit., p. 540, si la distribución entre 1893-95 desde Berlín fue una distribución general, se debe considerar la existencia de pliegos duplicados de estos de Ruiz y Pavón en Ginebra; también tienen, de 1950 y 1952 procedentes del British Museum (BM), en Saint Louis-Missouri (MO) y Nueva York (NY), así como en París (P) además de las de Dombey en las colecciones de Jussieu y Lamarck, Leningrado (LE) y Viena (W). Según W. Lack ${ }^{17}$, hay ejemplares en Berlín de esta expedición al Perú y Chile procedentes de Dombey que llegaron de París por mediación de Kunth en 1850 y de Humbert (1944?). También tienen en Berlín fragmentos y fotografías de los pliegos de Ruiz y Pavón prestados del (MA) Madrid después de 1929 por intermediación de Werdermann, para la realización de la Flora de Perú ${ }^{18}$.

16 McVaugh, R. (1987), p. 169.

17 LACK, H. W. (1979), "Die südamerikanishen Sammlungen von H. Ruiz und Mitarbeitern im Botanischen Museum Berlin-Dahlem», Wildenowia, 9, 177-198, pp. 181-182.

${ }^{18}$ Blanco Fernández de Caleya, P. (1989), «Identificación de los tipos en los herbarios históricos del Real Jardín Botánico de Madrid» en PESET, J. L. Ciencia, vida y espacio en Iberoamérica, 1, 207-234, pp. 219-220. 


\section{El herbario de Ruiz y Pavón en el (MA) del Real Jardín Botánico} de Madrid

En 1875, en tiempos de Colmeiro, el número de especies distintas que se encontraban en el herbario de esta colección eran 2.980. Hoy día está guardado en 165 cajas de cartón y 29 paquetes que aproximadamente contienen unos 8.000 pliegos, se supone que el número de tipos es alrededor de 1.800. Entre estas plantas hay ejemplares recogidos por Dombey que hizo intercambio con sus compañeros de expedición, por tanto en el herbario que llegó a París hay también plantas de las recogidas por Ruiz y Pavón.

\section{La Expedición del Reino de Nueva Granada}

La segunda expedición botánica organizada por Carlos III, la Real Expedición Botánica del Virreinato de Nueva Granada (1783-1808) fue gestada, gestionada y ejecutada por el sabio gaditano José Celestino Mutis (1732-1808) y continuada por su sobrino Sinforoso Mutis Consuegra hasta 1816 .

Es sin duda la de Mutis, la figura más sobresaliente entre la de los botánicos españoles que fueron a América en esta época. A los 25 años de edad obtuvo el título de Médico. Durante los años 1757-1760, compaginó la medicina con el estudio de la botánica en el Jardín Botánico de Migas Calientes, como discípulo de Miguel Barnades. Además se interesó por la astronomía, geografía y matemáticas, conocimientos que utilizó desempeñando distintas profesiones a partir de 1760 que desembarcó en América acompañando como médico al virrey de Nueva Granada, Messía de la Cerda, marqués de la Vega de Armijo.

Atraido por la naturaleza, observó y estudió la vegetación que le rodeaba. Así empezó a reunir materiales con los que producir una Flora de allí, a cuyo estudio se dedicó al ser nombrado en 1783, por Carlos III, primer botánico y astrónomo de la Expedición, tomando bajo su responsabilidad el estudio completo de los recursos naturales de aquel país, la promoción de su aprovechamiento y la educación de una juventud destinada a perpetuar esos intereses. Labor que cumplió sobradamente.

Mutis murió el 11 de septiembre de 1808 y aunque su sobrino Sinforoso se hizo cargo de la Expedición, las actividades decayeron poco a poco por las revoluciones independentistas del país. La casa donde guar- 


\section{PALOMA BLANCO FERNÁNDEZ DE CALEYA}

daban los materiales de la Expedición fue saqueada durante esas luchas y parte de las muestras vegetales fueron destrozadas o robadas. El general Morillo envió, en 1816, el herbario, los manuscritos y los dibujos de las plantas a Madrid. En total 104 cajas: 48 de herbario con unos 20.000 ejemplares aproximadamente, 14 de dibujos, una de manuscritos y el resto de semillas, muestras de maderas, minerales y dibujos de animales.

Al llegar a España se abrieron en presencia del Rey Fernando VII; los materiales de interés geológico y zoológico se trasladaron al Museo de Ciencias Naturales y el herbario, los manuscritos y las ilustraciones fueron depositados en el Real Jardín Botánico. Aunque Lagasca en 1817 fue encargado de la publicación de la Flora de Bogotá así como los manuscritos de Mutis sobre la quina, no le dieron ningún tipo de apoyo económico y no llegó a realizarse nada.

Otras plantas de Nueva Granada ya habían viajado a Europa en vida de Mutis, antes de ser oficial la Expedición. Sus receptores fueron Linneo y su hijo, en Suecia. Sobre ellas describieron y publicaron nuevos géneros y especies, cuyos nombres se incluyeron en Mantissa Plantarum (1771) y en Supplementum Plantarum (1781 [1782]). Muchas de estas plantas se encuentran en el herbario de Linneo (LINN) de la Sociedad Linneana de Londres. También en la visita que realizaron a Bogotá, Humboldt y Bonpland, en 1801, Mutis les cedió numerosas plantas que llevaron a Europa y que hoy forman parte del herbario histórico de Humboldt \& Bonpland (P-Bonpl) de París. Estas fueron descritas y publicadas en Plantae Aequinoctiales (1809), una obra dedicada por estos sabios a Mutis, y también en Nova Genera et Species Plantarum (1817-1825).

Mutis publicó muy poco. Sólo vieron la luz en 1769 algunos trabajos suyos en la Academia de Ciencias de Estocolmo; en 1784 describió su Pera arborea. En 1793 y 1794 empezó a publicar en el Papel Periódico de Santa Fe, una quinología, con el título El Arcano de la Quina. Más tarde se vio que las novedades que encerraban aquellos papeles eran sinonimias de otras especies publicadas por Linneo y Vahl. Su Memoria sobre Caryocar Amygdaliferum se publicó gracias a Cavanilles, que la incluyó en el tomo IV de sus Icones, en 1797.

Los 22 años que tardó la Expedición en aprobarse oficialmente, unido a su perfeccionismo o quizá inseguridad, que le hizo comunicar sus descubrimientos a otros botánicos europeos, hicieron que su gran empresa, por falta de publicación oportuna, no fuera valorada debidamente desde el punto de vista científico y aunque recibió honores en vida, su obra quedó como una gran recolección de plantas en una zona de Nueva Gra- 
nada, que es el herbario de la Expedición, una magnífica colección de láminas de esas plantas, y un archivo compuesto por cartas, diarios, y descripciones botánicas.

Aunque en 1881 el botánico colombiano José Triana fue autorizado por el Gobierno español para clasificar, determinar y publicar los dibujos y pinturas de la flora y fauna de Colombia, sólo pudo ordenar los dibujos y hacer un índice; no tuvo tiempo de asociar las ilustraciones con los numerosos ejemplares de herbario que permanecieron en las cajas originales sin tocarse hasta la primavera de 1932. Ellsworth Payne Killip, eminente botánico norteamericano y gran especialista en flora neotropical, visitó el Jardín Botánico de Madrid para examinar las primitivas colecciones de Ruiz y Pavón, Neé, Sessé y Mociño y los manuscritos de las exploraciones en América del Sur, así como las pinturas y dibujos de plantas de la Nueva Granada, hechas bajo la dirección de Mutis. Aprovechando esta visita, García Varela, entonces director del Real Jardín Botánico, le sugirió que se encargara del arreglo de la colección de Mutis, pactando un acuerdo con la Institución Smithsoniana para realizar una clasificación sistemática de los ejemplares, y posteriormente, la posible publicación de una Flora de Colombia, de acuerdo con los planes presentados por Mutis al Rey de España en 1783.

Killip fue pues, quien inició el estudio de las plantas mutisianas, que se guardaban intactas en este Jardín desde 1817. En Estados Unidos, Killip, con la colaboración de los botánicos del Instituto Smithsoniano y de otros centros americanos, C. V. Morton, C. Epling, N. L. Britton, W. Trelease, H. A. Gleason, S. F. Blake, B. L. Robinson, A. S. Hitchcock, L. B. Smith y otros, determinó el género o especie de buena parte de los 4.055 duplicados que habían llevado a Washington así como de los ejemplares únicos enviados en préstamo, que después devolvieron montados y determinados, y sobre los que se describieron novedades taxonómicas. Su papel fue muy importante para la flora mutisiana, al hacer asequibles a los monógrafos del siglo XX las plantas de su herbario, avanzando mucho en el conocimiento y valoración de ellas; incluso hoy día se siguen describiendo nuevas especies sobre este material ${ }^{19}$.

\footnotetext{
19 Blanco Fernández de Caleya, P. (1991), «Plantae mutisianae». Fontqueria 31, 153 216. 


\section{El herbario de Mutis en MA}

En tiempos de Colmeiro el número de especies distintas que se encontraban en el herbario de Nueva Granada eran alrededor de 6.000. Hoy la colección de Mutis tiene distintas numeraciones; la actualmente utilizada se inició en 1932 y hoy llega a la cifra de 6.490; en algunos casos hay números seguidos de letras, a, b, resultado de haberse mezclado distintas especies en un mismo pliego. Los duplicados se separaron y se intercambiaron con otros centros botánicos. Bajo la misma numeración se encuentran las plantas herborizadas por Mutis y los demás miembros de la Expedición, Eloy Valenzuela, Caldas, Sinforoso Mutis, Fray Diego García y Mejía. La localidad de muchas de las plantas es de los alrededores de Bogotá, y un número más reducido, de las cercanías de Mariquita. También hay ejemplares del Chocó, y de las montañas ecuatorianas.

La mayor serie de estas plantas se encuentra en el herbario MA. Como parte de estos ejemplares se enviaron en canje a Washington y se volvieron a separar a su vez para enviarlos a distintos especialistas, hoy existen también duplicados de Mutis en BM, COL, F, G, K, LINN, MEDEL, NY, P, S, SBT, ST, UPS, US. En esta colección de unos 20.000 ejemplares se pueden destacar 2.738 táxones diferentes, identificados por más de ciento cincuenta botánicos de todo el mundo; 306 de ellos corresponden a nombres basados en plantas mutisianas ${ }^{20}$.

\section{La Expedición al Reino de Nueva España}

Al coincidir la búsqueda de los manuscritos de Francisco Hernández, por parte de los eruditos Muñoz, Alzate y Bartoloche, con la propuesta del médico aragonés Martín de Sessé, desde Méjico, para inventariar la flora novohispana, en busca de sus aplicaciones terapéuticas para intentar reformar la sanidad, se aceleraron los trámites de la aprobación de una expedición a los territorios de Nueva España. Esta fue la Real Expedición de Nueva España (1787-1803), Méjico. Por Real Orden de 1786 se mandaba establecer en Méjico, un jardín botánico, su cátedra correspon-

\footnotetext{
20 Blanco Fernández de Caleya, P.; Del Valle Stervinou, A. (1991), Herbarium mutisianum seu catalogus plantarum a Novogranatensi Regia Legatione, sub Iosephi Caelestini Mutisii auctoritate lectarum. Adduntur aliae ab ipso lectae. Fontqueria 32, 1-173.

21 PUiG-SAMPER (1991), p. 42.
} 
diente y la organización de una expedición que debía formar los dibujos, recoger las producciones naturales e ilustrar y completar los escritos de Hernández. Para la dirección del futuro jardín y de la expedición se nombró a Martín de Sessé, a Vicente Cervantes, catedrático de botánica, a Juan Castillo, botánico de la expedición, a José Longinos Martínez, naturalista de la misma, y a Jaime Senseve, profesor farmacéutico agregado. Más tarde, en 1790, se unió José Mociño ${ }^{21}$. Durante ocho años, 17951803, la expedición recorrió una distancia de unas 3.000 leguas. Fruto de esos viajes fue un considerable herbario que vino a parar al Jardín Botánico de Madrid ${ }^{22}$. Las primeras actividades de la expedición comenzaron en 1787, en las zonas periféricas de la ciudad de Méjico. Al año siguiente se inició la primera campaña general ${ }^{23}$, desde el poblado de San Angel se hicieron excursiones hasta Yecapixtla y Xochitlán. Más tarde recorrieron las cercanías de Toluca. En la segunda campaña desde Méjico se llegó a Cuernavaca y Acapulco. En la tercera campaña, cuando se incorporaron Mociño y José Maldonado, se dirigieron a Michoacán y Sonora, se exploraron las zonas mineras de Querétaro, Guanajuato y Zacatecas y todo el recorrido hasta Guadalajara. De aquí se dividieron en dos gruos, uno formado por Mociño, Castillo y Echeverría, exploró la zona norte hacia Los Alamos, luego por la sierra de Tarahumara y la sierra de Tepehuanes hasta Aguascalientes; el otro grupo, Sessé, Maldonado y De la Cerda recorrieron las regiones de Sinaloa y Ostimuri. Longinos Martínez llegó a San Blas de Nayarit y a las dos Californias. A finales de 1792, Mociño, Maldonado y Echeverría se incorporaron a la expedición de Juan Francisco de la Bodega y Quadra al noroeste de América; de ella publicó Mociño sus Noticias de Nutka. Entre 1792-1794 se exploraron Puebla, Oaxaca, Córdoba, Veracruz, San Andrés Tuxtla, Tehuantepec y Tabasco. Solicitada una prórroga de dos años se pudo explorar Cuba, Puerto Rico y Guatemala. En Cuba coincidieron con la llegada de otra expedición, la del Conde de Mopox ${ }^{24}$.

\footnotetext{
22 Blanco Fernández de Caleya, P. (1987), "Los Herbarios de Sessé y Mociño» en el catálogo de la exposición La Real Expedición Botánica a Nueva España 1787-1803, Real Jardín Botánico de Madrid, CSIC, Madrid, pp. 253-260.

${ }^{23}$ Alvarez LÓPEZ, E. (1953), «Las tres primeras campañas de la expedición científica dirigida por Sessé y sus resultados botánicos». Anales del I. Botánico A. J. Cavanilles de Madrid XI (1), pp. 1-103.

${ }^{24}$ Puig-Samper, M. A. (1991), pp. 43.
} 
Además del grueso del herbario que llegó en 1803-1804, entre 1791 y 1816 se estuvieron recibiendo en el Jardín Botánico de Madrid semillas y propágulos, que crecieron y dieron lugar a plantas, que se pensó podían ser nuevas especies. Alrededor de 250 nuevos nombres se basaron en estas plantas crecidas en cultivo. Muchos de los nombres publicados por los botánicos españoles especialmente Cavanilles, son los primeros que se dieron a estas plantas, pero no fueron conocidos o aceptados por los botánicos europeos, quienes se afanaban en describir y nominar lo que ellos creían novedades.

Según $\mathrm{McVaugh}^{25}$, el principal objetivo que se percibe en Sessé y Mociño es la preparación y publicación de la Flora Mexicana. Para acompañarla con buenas ilustraciones los dibujantes de la expedición prepararon aproximadamente 1.800 dibujos botánicos. Por varios motivos, la mayor parte de ellos políticos, la ilustrada flora mexicana nunca se completó, ni siquiera el manuscrito; los dibujos originales se perdieron en Barcelona en 1820 y no aparecieron hasta 1979. Los distintos botánicos de la expedición murieron sin haber terminado su trabajo. Parte de los resultados fueron posteriormente publicados en Méjico.

No hay duda de que trabajaron juntos en el proyecto por lo menos durante los siguientes cuatro años a su regreso, tanto como pudo Sessé hasta su muerte en octubre de 1808. Después, durante la ocupación francesa (1808-1812) Mociño vivió en la pobreza y con muchos otros problemas. Luego de haber estado exilado algún tiempo, desgraciadamente murió en 1820 en Barcelona. Los dibujos, como antes dijimos, se perdieron a la muerte de Mociño, pero cerca de 255 duplicados realizados por los artistas originales y más de mil copias adicionales realizadas por otros artistas en Ginebra fueron estudiadas por botánicos europeos.

La actividad botánica estuvo de capa caída durante la dominación napoleónica, y esto pudo ser la causa por la cual el Herbario de la Expedición Botánica fuera descuidado durante varios años. Pavón aparentemente tuvo acceso a él en $1814^{26}$. Una «serie» de duplicados de estos ejemplares se sacaron entre 1814-1842 y fueron vendidos a colectores privados, que los distribuyeron por un gran número de herbarios europeos.

\footnotetext{
25 MCVAugh, R. (1987), pp. 168-170.

${ }^{26}$ McVAUGH, R. (1987), p. 168.
} 
David Don manifestó en 1824, que el herbario de Lambert incluía cerca de 2.000 plantas mexicanas (Lambert «A description of the genus Pinus, pág. 32-39). Probablemente la mayor selección de plantas mexicanas del herbario de Lambert se encuentre hoy en Ginebra (G), en el herbario Delessert y en el de Boissier. Ya hemos dicho que en el herbario del Instituto Botánico de Florencia, dentro de la colección que Webb recibió de Pavón entre 1826-1827, hay unas 2.345 plantas de Nueva España. Muchos botánicos europeos estudiaron y publicaron sobre estos «duplicados», por lo que se convirtieron en material muy valioso, sirviendo desde $1820-1880$ como tipo de los nombres de más de 500 nuevas especies, que se pueden añadir a aquellos nombres basados en los dibujos de Ginebra. Con el resurgir de la actividad botánica en Méjico desde 1870, más de la mitad de los manuscritos originales fueron publicados en aquel país entre 1887-1894, con el resultado de casi 1.500 nuevos nombres. Muchos de estos nombres fueron nuevos solamente desde el punto de vista de nomenclatura científica, pero pasados de fecha un siglo. La mayoría de las especies descritas en las floras póstumas habían sido ya publicadas mientras tanto por otros botánicos. Por todo esto, la publicación de Plantae Novae Hispaniae y Flora Mexicanae (1887-97) fue desafortunada desde el punto de vista botánico.

Aunque parte de los ejemplares fueron a parar a manos extrañas, el grueso se conservó en el Jardín Botánico de Madrid. También Mariano Lagasca, en los tiempos que estuvo en activo en el Real Jardín Botánico, tuvo acceso al herbario, y su letra aparece en bastantes etiquetas. Después de Lagasca, y quizá después de 1828, cuando la entresaca de duplicados que hizo Pavón pareció disminuir, el herbario estuvo sin tocarse alrededor de un siglo. Luego la colección entera fue temporalmente sacada del almacenaje y enviada a Chicago para ser estudiada e identificada por Paul C. Standley. Este botánico americano se encargó de que los ejemplares fueran montados y luego devueltos en 1964. Mientras las plantas estuvieron en el Field Museum (entonces conocido como The Chicago Natural History Museum), todas fueron fotografiadas. Aunque Standley se comprometió a estudiar y a publicar todo el contenido de este herbario, esto no llegó a realizarse, pero en 1958, otro botánico norteamericano, Roger McVaugh, se dedicó a la valoración de esta colección. Con la ayuda de más de cien especialistas en las familias de plantas tropicales, se identificó en términos modernos. Desde el punto de vista botánico el material es de gran valor, porque muchas veces no existe otra prueba directa de la identidad de una especie descrita en cualquiera de las dos floras póstumas de Sessé y Mociño. 


\section{PALOMA BLANCO FERNÁNDEZ DE CALEYA}

\section{El herbario de Sessé y Mociño en MA}

Según Colmeiro en 1875, constaba de 10 cajones con alrededor de 4.000 especies distintas. Las plantas de Sessé y Mociño recibidas mientras la expedición tenía lugar, se incluyeron en el Herbario General de entonces, junto con las de Cavanilles, Neé y Lagasca. El herbario que llegó entre 1803-180427, contenía por lo menos 20.000 especímenes, incluyendo duplicados. El lote que perdura hoy día en Madrid (MA) es la mitad; después de la entresaca de muchos duplicados que hizo Pavón, el número estimado es 6.000 o más ${ }^{28}$; también se extrajo un lote de 100 especímenes y 4.162 "fragmentos y unos pocos pliegos completos» para el Field Museum como intercambio por las identificaciones de P. C. Standley y por el montaje de la colección entera.

\section{La Expedición Malaspina}

La Expedición que dio la vuelta al mundo en 1789-1794, al mando del Capitán de Fragata Alejandro Malaespina, junto con el naturalista Antonio Pineda y los botánicos Luis Neé y Tadeo Haenke, intentó rivalizar con las grandes expediciones marítimas francesas e inglesas. Lo que nos interesa de ella, el herbario; se debe a los trabajos del botánico Neé.

Zarpó de Cádiz el 30 de julio de 1789 en la corbeta Atrevida al mando del Capitán de Fragata José Bustamante; con él iba José Guío, dibujante y disecador. Su compañero y jefe Pineda y el otro naturalista, Haenke, que los alcanzó en Valparaíso, iban en la Descubierta al mando de Malaespina ${ }^{29}$. "Neé compartiría todo el viaje de la Atrevida menos su escala en Acapulco, donde junto con Pineda y otros quedó en tierra para viajar a Méjico capital y recorrer el centro del Virreinato desde el 12 de abril hasta el 20 de diciembre de 1791, mientras las corbetas hacían viaje a Nutka y Monterrey; también se quedó en puerto en Sorsogón (Filipinas) el 3 de marzo de 1792 para ir por tierra hasta Manila, a donde llegó el 21 de junio; y en Talcahuano (Chile), el 3 de diciembre de 1793, para ir a Santiago de Chile y de ahí por Mendoza atravesando las Pampas, a Buenos

\footnotetext{
27 MCVAugh, R. (1990), p. 197.

28 MCVAUGH, R. (1987), p. 168.

29 Muñoz Garmendia, F. (1993), pp. 37-41.
} 
Aires y Montevideo, donde se reincorporó a las corbetas el 4 de junio de 1794, regresando a Cádiz el 21 de septiembre de 1794.

En el transcurso de la expedición, Neé se dedicó fundamentalmente a la recolección de plantas, en general sólo o en compañía de Pineda. Con Haenke no intentaron formar equipo ni sumar sus esfuerzos; cada uno herborizó por su lado e hizo las descripciones de las plantas por su cuenta sin apariencia alguna de cooperación; los herbarios marchaban separados en remesas hacia Cádiz.

Las plantas recolectadas por Neé durante la Expedición fueron acumulándose en la Real Aduana del puerto de Cádiz; parte fueron llevadas al Gabinete de Historia Natural de Madrid, a donde Neé las fue a recuperar a su vuelta. Entre los distintos paquetes, en una relación de «plantas colectadas en la expedición alrededor del mundo» dice haber un total de 15.990 y algunas otras más que no están en la lista.

Igual que hicieron los botánicos en las expediciones anteriores, Neé fue enviando semillas y otros materiales a la Península en el transcurso de su viaje. Las semillas que llegaron a Madrid en diferentes remesas, se entregaron al Real Jardín Botánico para su siembra. El primer dato correspondiente a los envíos de esta expedición al Jardín Botánico de Madrid, es acerca de la Ipomea pentaphyla (convolvulácea), que floreció en el Jardín en 1794. Entre los volumenes 4-6 de los Icones de Cavanilles (1797-1801) y en sus Descripciones (1801-1802), hay un montón de nuevas descripciones con referencias a semillas traídas por Neé y luego cultivadas en el Jardín Botánico. En cuanto a los trabajos escritos por Neé, parece ser que se perdieron; existen descripciones formalmente correctas aunque sin nombre científico, pero son pocas en relación con el número de las plantas herborizadas.

Neé desconfiaba de Haenke, el otro botánico que formó un herbario paralelo al que oficialmente se iba remitiendo a Cádiz; el herbario que Haenke enviaba a la misma ciudad peninsular, por medio de filiales americanas, se guardaba en los almacenes de una empresa gaditana y allí estuvo hasta que fue a parar, tras algunas vicisitudes, al Museo Nacional de Praga, donde aún se encuentra ${ }^{30}$. El herbario de Haenke, reunía 15.000 ejemplares (4.000 especies o variedades). Llegó a Praga en siete cajas que contenían 84 paquetes de plantas, maderas, raíces y resinas

30 IBAÑEZ, M. ${ }^{\text {a }}$ V. (1989), "Nuevas aportaciones a la investigación haenkeana» en L. Higueras (ed.), La botánica en la Expedición Malaspina, p. 50. 


\section{PALOMA BLANCO FERNÁNDEZ DE CALEYA}

enviadas desde Cádiz en 1794. Estas plantas sirvieron a K. B. Presl para editar, en 1825 y 1835 , los dos tomos de Reliquiae haenkeanae. Seguramente entre ellas están algunas plantas que Neé dio a Haenke.

\section{El herbario de Neé en MA}

De acuerdo con Colmeiro (1875), estaba incluido en el Herbario General, junto con el de Cavanilles, parte del de Sessé y Mociño, Lagasca, Rodríguez y Salcedo, etc.; en total 14.000 especies, de las cuales una tercera parte eran nuevas ${ }^{31}$. "Formó un herbario copioso alrededor de 12.000 números, con plantas de medio mundo, muy variadas y de sitios recién descubiertos como Nueva Holanda y Vaovao. La importancia de este herbario radica en que cayó en manos de Cavanilles, que lo ordenó adecuadamente; luego en las de Lagasca, con resultados menores pero no desdeñables y pronto salió al extranjero con los múltiples intercambios que mantenían los botánicos hispánicos, cayendo sus duplicados en manos de J.E. Smith, O.P. Swartz, C. L. Willdenow, C. S. Kunth y A. P. De Candolle por citar algunos pocos de los que publicaron nuevas especies fundadas sobre material de Neé. La importancia de estos envíos y de semillas es grande ya que fue la manera de distribuir nuevas plantas en Europa».

\section{La Expedición a Cuba del conde de Mopox}

Otra expedición con resultados para los herbarios del Real Jardín Botánico de Madrid fue la de la Real Comisión de Guantánamo, en Cuba, dirigida por el Conde de Mopox, entre 1796-1802; llevaba a Baltasar Manuel Boldo, como encargado del estudio de la vegetación de la isla. Boldo, médico del Real Jardín, fue nombrado botánico de esta expedición, cuyo objetivo principal era militar y donde la botánica fue un apéndice de un «proyecto de ordenación ambiental». Tras herborizar la parte occidental de la isla se le agregó a la expedición José Estévez, quien a su muerte, en 1799, se encargaría de ordenar y terminar las descripciones. En total un manuscrito de 742 folios, con descripciones de plantas de La

31 Muñoz Garmendia, F. (1993), p. 41. 
Habana y sus alrededores, clasificadas según las 24 clases de Linneo, excepto la séptima. Los manuscritos, junto con las 66 láminas de plantas dibujadas por José Guío, son la primera aportación importante de la botánica cubana.

\section{El herbario de Boldo en MA}

Llegó a España a principios del siglo XIX con José Estévez; constaba de cuatro cajones de plantas secas, alrededor de 2.000 ejemplares, que venían junto con las descripciones. Según Cavanilles, muchas de estas plantas se encontraban en tan buenas condiciones de conservación, que pasaron a formar parte inmediatamente del Herbario General del Real Jardín Botánico. Las fanerógamas se guardaron en el herbario general antiguo, hoy incluido en el herbario general; los ejemplares que Cavanilles utilizó para describir nuevas especies se encuentran en el herbario de tipos de Cavanilles; las criptógamas se guardaron en su lugar correspondiente del herbario de Criptogamia. En el herbario de Boldo existen especies no cubanas, procedentes del viaje que realizó a América del Norte, durante el desarrollo de la Comisión o producto de intercambios. Las localidades citadas son Virginia, Marylandia, Baltimore, Nueva Orleans, Panamá, Jardines botánicos de Washington y Hamilton y otras cubanas que no figuran en el manuscrito del Real Jardín Botánico ${ }^{32}$ :

\section{Herbarios de Filipinas}

Además del ya mencionado Neé, podemos destacar otros botánicos españoles que recolectaron en Filipinas en el siglo XIX, aunque no con motivo de expediciones científicas organizadas como las anteriores.

El agustino, explorador y botánico Francisco Manuel Blanco (17781845), se dedicó en Filipinas al estudio de las plantas y llegó a publicar en Manila la Flora de Filipinas según el sistema sexual de Linneo (1. ${ }^{a}$ ed. 1837, 2. ${ }^{a}$ ed. 1845, 3. ${ }^{a}$ ed. 1877-1883), esta última edición aumentada con

32 Blanco, P.; Morales, R. (1990), «Las plantas de Boldo y Estévez en el Herbario MA" in Cubensis Prima Flora, Fontqueria 29, pp. 185-188, p. 185. 


\section{PALOMA BLANCO FERNÁNDEZ DE CALEYA}

el manuscrito inédito del P. Fr. Ignacio Mercado y las obras de P. Fr. Antonio Llanos ${ }^{33}$. En el herbario MA se conservan plantas filipinas de los padres Blanco y Llanos. En época de Colmeiro eran 400 especies. En 1955, según la conservadora del herbario, Dra. Elena Paunero, se encontró un paquete con 260 plantas filipinas manuscritas por el padre Blanco. En el herbario de Leiden (L), el Dr. Quisumbing encontró en 1958 siete ejemplares de estas plantas filipinas de Blanco.

El viaje de Juan de Cuellar a Filipinas (1785-1806?) ha recibido poca atención por parte de los historiadores de las expediciones científicas del siglo XVIII ${ }^{34}$. Juan de Cuellar, botánico y farmacéutico, fue contratado por la Real Compañía de Filipinas para fomentar y dirigir el cultivo de plantas útiles para el desarrollo agrícola y comercial, principalmente el cultivo y cuidado de las plantaciones de canelos. Por Real Orden del 19 de noviembre de 1785 se le otorgó el título de «Botánico Real sin sueldo..., para que lleve adelante la formación de herbarios, y colecciones de productos naturales, describiendo y delineando puntualmente las plantas que se encuentren en aquellos mis fértiles dominios, para enriquecer mi Gavinete de Historia Natural y Jardín Botánico de la Corte...». Esta Real Orden la acometió ampliamente con la creación de dibujos de plantas y animales de la isla. Las láminas de las plantas han sido encontradas recientemente por la investigadora Belén Bañas, en el Archivo de Real Jardín Botánico de Madrid; del herbario aún no hay noticias de su paradero. En 1789 escribió la Descripción del árbol que produce la canela de Manila, según se ve en el Memorial literario de Madrid correspondiente a julio de $1793^{35}$.

Sèbastián Vidal y Soler (1842-1889) llegó a Filipinas en 1871 como inspector de montes y luego, en 1876, fue jefe de la Comisión de la Flora Forestal de Filipinas. De 1878-1889 fue director del Jardín Botánico de Manila. Además de sus múltiples publicaciones sobre la Flora Forestal Filipina, donde describe un buen número de especies nuevas, se con-

33 Colmeiro, M. (1858), p. 205.

34 BAÑAS, B. (1988), «Identificación de las láminas de Juan de Cuellar existentes en el Real Jardín Botánico de Madrid». Anales Jard. Bot. Madrid 45, p. 357.

35 Colmeiro, M. (1858), p. 182. 


\section{LOS HERBARIOS DE LAS EXPEDICIONES CIENTIIFICAS ESPAÑOLAS AL NUEVO MUNDO}

serva en el herbario del Real Jardín Botánico de Madrid (MA) una colección de unas 8.000 especies (25.000? con los duplicados), muy bien documentada y revisada por el Dr. Quisumbing a partir de 1954. También hay plantas de Vidal en los herbarios FI, G, K, L, P y PNH. El lote principal que se encontraba en Manila lo destruyó un fuego de 1897.

\section{La Comisión Científica al Pacífico (1862-1866)}

La última y verdadera expedición española a América fue la Comisión Científica al Pacífico (1862-1866), que llevaba como botánico a Juan Isern. Los objetivos básicos de la misión eran: para los oficiales de marina de la Escuadra, «recoger datos sobre observaciones astronómicas y geográficas», y para los científicos civiles, «recoger seres y plantas de la naturaleza para enriquecer con nuevas especies las colecciones existentes y fomentar la propagación y aclimatación de otros útiles a la vida del hombre y provechosos para la aplicación de la Ciencia» ${ }^{36}$. El botánico Juan Isern, fue el más diligente; su herbario cuantioso y sus descripciones se hallan en el Real Jardín Botánico de Madrid. Al crearse la sección de Flora Tropical del Jardín Botánico de Madrid, en 1932, esta colección se incorporó a esa sección y su estudio fue una de las tareas que se impuso Don José Cuatrecasas Arumi. En 1935 publicó las especies recogidas en Ecuador, Bolivia y Perú correspondientes a las familias de las compuestas, gesneriáceas, gramíneas, cariofiláceas, berberidáceas y pocas más, constituyendo una primera serie Plantae Isernianae, que desgraciadamente no se continuó. En esta primera se describen 58 especies, variedades, formas y combinaciones nuevas.

\section{El herbario de Isern en MA}

Las excursiones de Isern por Brasil, Argentina, Chile, Perú, Bolivia y Ecuador y un descenso por el Amazonas, se reflejan en más de 8.600 distintos números de ejemplares que envió a España, cuidadosamente preparados y etiquetados con todo tipo de detalles. Actualmente se encuen-

\footnotetext{
36 Puig-SAmper, M. (1988). Crónica de una expedición romántica al Nuevo Mundo: La Comisión científica del Pacífico (1862-1866), 1-459.
} 


\section{PALOMA BLANCO FERNÁNDEZ DE CALEYA}

tra en estudio y luego volverá a formar parte del Herbario General. De momento 300 especies corresponden a material tipo ${ }^{37,38}$.

\section{Epílogo}

Aunque la mayor parte de los expedicionarios a finales del siglo XVIII y principios del siglo XIX, tenían la idea de publicar sus distintas floras al terminar el período expedicionario y a ello se dedicaron al volver definitivamente de sus viajes, no tuvieron mucha suerte por los cambios políticos que ocurrieron al comenzar el XIX. Sin embargo, antes de su vuelta a la Península, las plantas procedentes de las semillas que enviaron desde sus distintos países de expedición, Perú y Chile, Nueva España y la de la vuelta al mundo, se cultivaron, crecieron y fueron estudiadas en Madrid por los botánicos que se encontraban entonces en el Real Jardín, Gómez Ortega, Cavanilles, y más tarde, Lagasca. Entre ellas se encontraron muchos géneros y especies nuevas. Estas plantas remitidas desde América se replicaron a su vez en Madrid para enviarlas como intercambio a los distintos centros de Europa. Este material vivo distribuido desde el Real Jardín Botánico fue una importante contribución a la horticultura y al conocimiento de las flores tropicales americanas. También hay que recordar las especies que viajaron desde América a otros países de Europa como las de Mutis a Suecia y a Francia, las de Dombey a Francia, las de Haenke a Praga, sin olvidar la gran cantidad de ellas que salieron de España distribuidas por José Pavón y que también dieron lugar a nombres nuevos.

En el siglo XX, destacaremos la figura de Don Ignacio Bolívar que desde 1921 hasta 1930, se hizo cargo de la dirección del Real Jardín Botánico. Aunque era entomólogo, fue el que influyó en la Junta para Ampliación de Estudios para que se revitalizara el estudio de las floras americanas a base de las antiguas colecciones, creando en el Jardín la sección de

\footnotetext{
37 Blanco Fernández de Caleya, P. (1988), «Los herbarios de Mutis e Isern». Asclepio 40 , pp. $359-373$.

38 Blanco Fernández de Caleya, P. y Puig Samper, M. A. (1995), «Plantas de R. A. Philippi (1808-1904) en el Herbario de la Comisión Cientìfica al Pacífico (1862-1866) del Real Jardín Botánico de Madrid». Anales Jard. Bot. Madrid 53(1), 55-99.
} 
Flora Tropical. Por ello, el 14 de marzo de 1933, fue nombrado jefe de esa sección el Dr. José Cuatrecasas Arumi, con encargo de conservar, organizar y estudiar las colecciones tropicales del herbario, pues los otros conservadores del Jardín dedicados a la flora española y norteafricana, se encargaban del Herbario General. Luego director del Jardín Botánico hasta 1938, es uno de los científicos españoles que más ha contribuido a revalorizar la labor de nuestros botánicos de finales de los siglos XVIII y XIX.

Trató desde el primer momento de establecer contacto con otras Instituciones botánicas y gracias a ello se pudieron enviar estas colecciones a Berlín, Ginebra, Washington, Chicago..., donde fueron estudiadas y actualizadas por los botánicos especialistas. Debido a este intercambio con otros centros botánicos, duplicados de plantas de Mutis, de Ruiz y Pavón, de Sessé y Mociño, de Isern y de Cuatrecasas también salieron de España en el presente siglo y hoy se encuentran en diversos herbarios de Europa y América.

Todas estas plantas y los datos que hay sobre ellas documentan la existencia de las especies en el tiempo y en el espacio. Muchas plantas no podrán recolectarse de nuevo debido a la destrucción actual del medio ambiente. Con su estudio se podrán establecer estrategias para el reconocimiento y entendimiento de la crisis de la biodiversidad, esencial para la conservación, gestión y uso de nuestros ecosistemas. 